\title{
Lothar Kettenacker
}

\section{Kommentar zu III. Die Herausforderung des Nationalsozialismus und die Appeasement-Politik}

Zweifellos war die Friedensbewahrung das erklärte Ziel der westlichen Staatsmänner in den 1930er Jahren, und ohne Zweifel sind sie damit gescheitert. Aber kann man in der Zwischenkriegszeit wirklich von einem Friedenszustand sprechen oder von einem Friedenssystem, das von den U.S.A. nie ratifiziert worden ist, von der britischen Regierung bald als revisionsbedürftig angesehen und von Berlin grundsätzlich in Frage gestellt wurde? Viel spricht für den von D. C. Watt bemühten Vergleich mit dem 30jährigen Krieg. Gemeint ist, daß die Epoche von 1914-1945 als eine Einheit gesehen werden muß und als eine Art europäischer Bürgerkrieg interpretiert werden kann'1. Demokratie, Faschismus und Bolschewismus standen sich wie zu Zeiten der Religionskriege als antagonistische Prinzipien gegenüber. Und es sah für viele so aus, als habe die liberale Demokratie als Gesellschaftsmodell ausgedient. Dieser Kommentar soll die britischen und französischen Antworten auf die Herausforderung des Nationalsozialismus, der mit Abstand gefährlichsten Variante des europäischen Faschismus, miteinander in Vergleich setzen.

Es erweist sich - der Beitrag von Alexander macht dies wiederum deutlich ${ }^{2}$-, daß die Historiographie in beiden Ländern von unterschiedlichen Bezugspunkten ausgeht. Die britische Geschichtsschreibung kann sich von dem Trauma der Appeasementpolitik nicht freimachen. Die Anfänge der Appeasementforschung waren jedoch von Beweggründen motiviert, die heute ihre Gültigkeit weitgehend verloren haben: Die Verständigung des Westens im Zeichen des Kalten Krieges auf die gemeinsame Verantwortung für "the rake's progress“, also Hitlers ungehinderte Vorbereitung des Krieges, sodann die innerbritische Vergangenheitsbewältigung, ausgelöst durch Churchills Memoiren und seine Behauptung, der Krieg sei

${ }^{1}$ Donald C. Watt, The European Civil War, in: Wolfgang J. Mommsen und Lothar Kettenacker (Hrsg.), The Fascist Challenge and the Policy of Appeasement (London 1983) 3-21. 2 Vgl. Martin S. Alexander, The Franco-German Balance of Power in the Appeasement Era: Perspectives from the Hôtel Matignon and the French Army, in diesem Band, $159 \mathrm{ff}$. 
leicht zu verhindern gewesen ${ }^{3}$. Bei französischen Historikern hat man es mit einem anderen Trauma zu tun, das heute erkenntnisleitenden Charakter hat: Es ist die eklatante Niederlage im Frühsommer 1940. Auf eine englische Formel gebracht lautet der Unterschied: „Honour to be saved or blood to be spared“. Diese Disposition, die Suche nach Ursachen des nationalen Desasters, führt in beiden Fällen nur allzu leicht zu der Frage nach den Schuldigen, "the guilty men"4. In Großbritannien saß lange Zeit Chamberlain, der sich, da früh verstorben, selbst nicht verteidigen konnte, auf der Anklagebank. Erst nach Freigabe der Akten ist ihm von der Forschung ein gewisses $\mathrm{Maß}$ an Gerechtigkeit widerfahren ${ }^{5}$. Es wurde deutlich, wie eng sein Handlungsspielraum im Grunde war, sah er sich doch auch durch die Einstellung der Stabschefs und der Dominions 1938 in seiner Option für Verhandlungen bestätigt. Churchill hatte vor dem Krieg für eine noch rigorosere Aufrüstung plädiert, sodann für eine grand alliance, möglichst unter Einbeziehung der U.S.A. und der Sowjetunion. Aber ob er dies als Premierminister auch wirklich zustande gebracht hätte, und wenn ja, ob dies Hitler abgeschreckt hätte, ist ungewiß. Die Herausstellung Barthous als des zu früh verstorbenen, potentiellen Retters Frankreichs, entspricht im Grunde der überwundenen Anklagehaltung gegenüber Chamberlain. Der Beitrag von Martin Alexander vermittelt den Eindruck, als habe sich die französische Historiographie von dieser personenbezogenen Betrachtungsweise noch nicht gelöst $t^{6}$. Man begibt sich damit auf die Argumentationsebene Hitlers, der den westlichen Demokratien vorwarf, $\mathrm{da} ß$ es ihnen an entschlossenen Staatsmännern fehle. Im November 1937 versicherte er dem inneren Führungszirkel, „daß mit hoher Wahrscheinlichkeit England, voraussichtlich aber auch Frankreich die Tschechei im Stillen abgeschrieben und sich damit abgefunden hätten, daß diese Frage eines Tages durch Deutschland bereinigt werde"

Der Zusammenbruch des Staatensystems, das durch Versailles und den Völkerbund geschaffen werden sollte, und die Rückkehr zu Bilateralismus und traditionellem Allianzsystem hätte einen völligen Bewußtseinswandel in den westlichen Demokratien zur Folge haben müssen. Das war bekanntlich nicht der Fall. In Großbritannien war die Gesellschaft nicht für eine erneute Intervention auf dem Kontinent zu haben, in Frankreich verbreitete sich die Maginot-Linien-Mentalität. Während der geistige Militarismus in Deutschland nie eine wirkliche Demobi-

${ }^{3}$ Siehe Winston S. Cburchill, The Second World War, Bd. 1: The Gathering Storm (London 1948) 14.

4 Titel einer 1940 erschienenen Satire gegen die Appeasers der Vorkriegszeit. S. Paul Addison, The Road to 1945. British Politics and the Second World War (London 1975) 110.

${ }^{5}$ Sehr ausgewogen, d.h. nicht übertrieben revisionistisch: Robert A. C. Parker, Chamberlain and Appeasement (London 1993). Siche auch den Beitrag des Vf.: Diplomatie der Ohnmacht. Die gescheiterte Friedensstrategie der britischen Regierung vor Ausbruch des Zweiten Weltkriegs, in: Wolfgang Benz und Hermann Graml (Hrsg.), Sommer 1939. Die Großmächte und der Europäische Krieg (Stuttgart 1979) 223-79.

$6 \mathrm{Vgl}$. Alexander, in diesem Band.

7 Hoßbach-Protokoll vom 5. 11. 1937, zit. nach Bernd-Jürgen Wendt, Großdeutschland. Außenpolitik und Kriegsvorbereitung des Hitler-Regimes (München 1987) 199; s.a. 11-37. 
lisierung erfahren hatte, war die Bevölkerung im Westen nach den und dem Massenschlachten im Weltkrieg den Krieg gründlich leid. Dies erklärt auch, warum die westlichen Staatsmänner den äußersten Kriegswillen Hitlers lange Zeit nicht wahrhaben wollten, waren doch die nachvollziehbaren Ziele Deutschlands ohne Krieg zu erreichen. Umso mehr Anerkennung verdient es, daß den Einschüchterungsversuchen des deutschen Diktators am Ende kein Erfolg beschieden war. Vielleicht war ja der Krieg gar nicht zu verhindern, angesichts der Entschlossenheit, mit der Hitler darauf zusteuerte, ohne daß er durch internen Widerstand daran gehindert worden wäre. Er wußte nur zu gut, daß seine imperialen Träume ohne Krieg nicht zu verwirklichen waren ${ }^{8}$. Aber den Krieg, den er sich gewünscht hatte, eine Sequenz von Blitzkriegen, hat er nicht bekommen.

Gewiß hatte Hitler sich mit seinen Nahzielen, der hemmungslosen Aufrüstung, der Rheinlandbesetzung, der Annexion Österreichs durchsetzen können. Aber schon München, als Inbegriff westlicher Nachgiebigkeit gegenüber dem Diktator diskreditiert, war aus Hitlers Sicht kein voller Erfolg. Denn letztlich hatte er das einzige Ziel, das er in den 30er Jahren mit rigoroser Konsequenz verfolgte, nicht erreichen können, nämlich das Desinteresse der westlichen Demokratien an Osteuropa, die „freie Hand“ für die Landnahme im Osten, um die angebliche Raumnot des deutschen Volkes zu beseitigen. Unmittelbar vor Kriegsausbruch äußerte sich Hitler gegenüber Carl Jakob Burckhardt: „Alles, was ich unternehme, ist gegen Rußland gerichtet; wenn der Westen zu dumm und zu blind ist, um dies zu begreifen, werde ich gezwungen sein, mich mit den Russen zu verständigen, den Westen zu schlagen und dann nach seiner Niederlage mich mit meinen versammelten Kräften gegen die Sowjetunion zu wenden." ${ }^{9}$ Auch nach Abschluß des deutsch-sowjetischen Nichtangriffspaktes waren die westlichen Demokratien nicht bereit, ihm kampflos das Feld zu überlassen. Hitlers $\mathrm{Haß}$ auf die von ihm als Haßgegner apostrophierten westlichen Demokratien zeigt zu deutlich, daß er mit seiner Divide-et-impera-Politik, ungeachtet aller Teilerfolge, gescheitert war. Seine ganze Diplomatie war darauf gerichtet, einen Zwei-Fronten-Krieg wie im Ersten Weltkrieg zu vermeiden. An Bismarck hatte er vor allem bewundert, daß er die Vormacht in Deutschland und dann die Reichseinheit auf dem Schlachtfeld ohne die Intervention anderer Mächte erzwungen hatte ${ }^{10}$. Die Genialität des groBen Staatsmanns bestand in seinen Augen darin, die günstigste Konstellation für den Kriegsbeginn zu schaffen, für das „blitzartige Losschlagen“, wie er es nannte. Nach dem Eintreffen des britischen Ultimatums am 3. September 1939 soll er in seiner neuen Reichskanzlei ratlos und wie versteinert dagesessen haben ${ }^{11}$. Fortan

${ }^{8}$ Vgl. ebd. 197: „Zur Lösung der deutschen Frage könne es nur den Weg der Gewalt geben, dieser niemals risikolos sein“.

9 Carl Jacob Burckbardt, Meine Danziger Mission (München 1960) 348.

10 Vgl. Hitlers Zweites Buch, hrsg. von Gerhard L. Weinberg (Stuttgart 1961) 108.

11 Vgl. Paul Schmidt, Statist auf diplomatischer Bühne 1923-1945 (Bonn 1950) 463f. Laut Picker soll Hitler später bei Tisch gesagt haben, daß er „zu keiner Zeit mit einer britischen Kriegserklärung" gegen Deutschland wirklich ernsthaft gerechnet habe. S. Henry Picker, Hitlers Tischgespräche im Führerhauptquartier (Wiesbaden 1983) 50. 
sollte er nur noch militärische Siege erringen, keine politischen. Keiner dieser Siege, erst gegen Polen, dann gegen Frankreich, dem jedesmal eine Friedensoffensive folgte, brachte ihn seinem eigentlichen Ziel näher.

Seine wahren Absichten und operativen Überlegungen hat Hitler immer nur einem kleinen Kreis der obersten Wehrmachtsführung und dem Reichsaußenminister anvertraut, also nur jenen, auf deren Kooperation er angewiesen war. Man fragt sich heute, ob denn in diesem kleinen Kreis wenigstens Klarheit darüber bestand, welche Absichten der „Führer" langfristig verfolgte, wenn er von der „Bodenpolitik der Zukunft“ oder der „Raumnot des deutschen Volkes“ sprach. Der schiere Atavismus einer aus dem Mittelalter ins 20. Jahrhundert transplantierten Ostkolonisation konnte stets bloß als visionär verstanden werden. Man wird an die antisemitische Polemik Hitlers erinnert, an die sich steigernden Haßtiraden gegen die Juden, die auch nicht die mörderischen Konsequenzen vorausahnen lieBen. Wie sollte da in den Kanzleien des Westens, dort, wo common sense den Ton bestimmte, Klarheit darüber geherrscht haben, mit wem man es in Wahrheit zu tun hatte? Hat nicht noch Lloyd George Hitler als den George Washington des neuen Deutschland gepriesen ${ }^{12}$ ? Die Mutmaßungen über die letzten Absichten Hitlers, die im westlichen Ausland angestellt wurden ${ }^{13}$, orientierten sich verständlicherweise an den Traditionslinien des deutschen Nationalismus vor 1914: die verwirklichung des Nationalstaates unter Einschluß möglichst aller Volksdeutschen sowie Kolonialbesitz in Übersee. Daß diese Ziele als legitim angesehen wurden, läßt die Reaktion auf den Anschluß Österreichs und das Münchener Abkommen erkennen. Woran man Anstoß nahm, war das die Interessen der europäischen Staatengemeinde mißachtende Vorgehen des deutschen Diktators. Daher die wiederholt vorgebrachte Forderung nach einem die Ziele Hitlers begrenzenden general settlement. Form und Inhalt der Politik kamen sich in ihrem Bedeutungsgehalt so nahe wie nie zuvor. Es ging einmal um die Grenzen und den Charakter des deutschen Nationalstaates. Zum anderen war die Vorgehensweise Hitlers ein entscheidendes Kriterium: Das Überschreiten der Grenze mit Waffengewalt bezeichnete das rien ne va plus. Mit dem Einmarsch in Prag im März 1939 hat Hitler beide Grenzen überschritten, freilich ohne auf Widerstand zu stoßen. Die Besetzung Böhmens und Mährens gehörte noch zu den „bloodless victories“, die Halifax nicht weiter akzeptieren mochte ${ }^{14}$. So gesehen war mit der Garantie Polens nicht nur eine abschreckende Wirkung bezweckt, sie diente ebenso dazu, den casus belli genau zu definieren, um sich selbst in die Pflicht zu nehmen ${ }^{15}$. Gemäß der römisch-imperialen Maxime si vis pacem para bellum suchte die britische Regierung

12 Vgl. Angela Schwarz, Die Reise ins Dritte Reich. Britische Augenzeugen im nationalsozialistischen Deutschland 1933-39 (Göttingen 1993) 64.

${ }^{13}$ Siehe Philipp W. Fabry, Mutmaßungen über Hitler (Düsseldorf 1969) 173-222.

14 Frederick Earl of Birkenbead, The Life of Lord Halifax (London 1965) 434.

15 Zur britischen Polenpolitik: Simon Newman, March 1939. The British Guarantee to Poland (Oxford 1976); Anita Prazmowska, Britain, Poland and the Eastern Front, 1939 (Cambridge 1987) bezweifelt überhaupt die Entschlossenheit Londons, Polen im Ernstfall zu verteidigen. 
den Frieden zu erhalten, indem sie sich auf den Krieg vorbereitete, allerdings ebenso wie die französische Regierung nur auf den Verteidigungskrieg.

Der herkömmlichen Meinung zufolge wurde der Frieden verspielt, weil die Rüstung nicht genug forciert wurde und im strategischen Ansatz zu defensiv war. Tatsächlich entsprachen jedoch die Rüstungsanstrengungen den wirtschaftlichen Möglichkeiten innerhalb des politisch vorgegebenen Rahmens der westlichen Demokratien. Dekadent war Frankreich nur aus der Sicht des nationalsozialistischen Deutschland und angesichts der von letzterem ausgehenden Bedrohung. Nach München haben es die Demokratien zwar an realen Vorbereitung für den Ernstfall nicht fehlen lassen, wohl aber an einer propagandistischen Zurschaustellung der Entschlossenheit und an klaren Worten an die Adresse der deutschen Generalität. $\mathrm{Zu}$ sehr bestimmte in London das innenpolitische bzw. imperiale Bedingungssystem den Kurs der Politik, nach dem Motto business and diplomacy as usual. Für Großbritannien war das letzte Friedensjahr entscheidend, um die Dominions für den Kriegseintritt und um die Luftschlacht im Jahr darauf zu gewinnen. Selbst der Demütigungsprozeß, dem der Westen ausgesetzt war, hatte andere als die von Hitler beabsichtigten Folgen: Er diente der psychologischen Kriegsvorbereitung. Diese war von einem weit höheren Maß an Rationalität gekennzeichnet als die Friedenserhaltung, der mindestens zwei unbekannte Größen zugrunde lagen, nämlich die Absichten Hitlers und Stalins. In beiden Fällen unterlagen die Einschätzungen gravierenden Fehlspekulationen, suchte man doch auf der Grundlage des für das eigene System maßgebenden common sense die Interessen des ideologischen Gegners zu antizipieren und zu befriedigen. Ziebura spricht hier zu Recht von "grundsätzlicher kognitiver Inkompatibilität gegenüber einem Phänomen, das allen eigenen Denkgewohnheiten und Wertmustern so radikal widersprach“16. Und Karl Rohe erklärt damit die „Inkorporationsstrategie der Appeasementpolitiker, die an vermeintlichen Gemeinsamkeiten mit dem gesellschaftlichen Gegner anknüpft" 17 . In Frankreich trugen autoritäre und korporatistische Ideen dazu bei, den Nationalsozialismus als populistische Variante des traditionellen deutschen Nationalismus zu interpretieren. In Großbritannien lieferte das Vorbild des Sterling-Blocks, der in vieler Hinsicht an das informal empire früherer Zeiten erinnerte, ein Modell, um geopolitischen Aspirationen des Deutschen Reiches in Ost- und Südosteuropa entgegenzukommen. Es erschien sinnvoll, daß Deutschland über seine Siedlungsgrenzen hinaus politischen und wirtschaftlichen Einfluß ausüben wollte, Rohstoff- und Absatzmärkte für sich zu erschließen suchte. Die Sondierungen des britischen Außenministers im Herbst 1937 lassen vermuten, daß solche Interessen Berlins in einem general settlement durchaus Berücksichtigung finden konnten ${ }^{18}$.

16 Gilbert Ziebura, Determinanten der Außenpolitik Frankreichs, 1932-1939, in: Karl Robe (Hrsg.), Die Westmächte und das Dritte Reich 1933-1939. Klassische Großmachtrivalität oder Kampf zwischen Demokratie und Diktatur? (Paderborn 1982) 152.

17 Ebd. 196.

18 Vgl. Wendt, 118 f. sowie ADAP, Serie D, Bd. 1 (Göttingen 1956) Dok. Nr. 31. 
Mit anderen Worten, selbst die Vorstellung einer „freien Hand“ in Osteuropa konnte von Hitler so und von den westlichen Demokratien ganz anders verstanden werden: zum einen Ausdehnung der Siedlungsgrenzen, zum anderen Vorherrschaft ohne territoriale Veränderungen. Und selbst wenn Hitler von Kolonisation im Sinne des Blut- und Bodenmythos eines Richard Walter Darré träumen mochte, war ihm von seiner politischen Entourage nicht beizubringen, daß es im 20. Jahrhundert andere, lukrativere Formen ökonomischer Ausbeutung und politischer Hegemonie gab? Anders formuliert, in Anlehnung an einen sehr prägnanten Buchtitel ${ }^{19}$ : Auch die Entente-Mächte konnten sich mit Großdeutschland abfinden, solange es nicht zum Krieg kam, weder zum großen noch zum kleinen. Ich glaube, daß hier Hoffnungen und Mißverständnisse eine merkwürdige Symbiose eingegangen sind. Ihren Höhepunkt erreichte die Konfusion mit der deutschfranzösischen Vereinbarung vom 6. Dezember 1938, einen Monat nach der den Charakter des Regimes bloßstellenden Kristallnacht ${ }^{20}$. Das Hauptmerkmal der französischen Politik während der sechs Monate nach München sei „ambiguity“ gewesen, schreibt Adamthwaite in einem dieser Begegnung gewidmeten Artikel. Die Allianzen mit der Sowjetunion und Polen wurden öffentlich nicht in Frage gestellt. "None the less German predominance in the east was tacitly recognised. “21 Aber, was hieß schon „predominance“? Es hieß: „Informal Empire Yes, formal Empire No." Es gibt keine Anhaltspunkte dafür, daß Hitler solchen Gedankengängen zugänglich war, daß er mit dem Begriff informal empire etwas anfangen konnte. Dazu war sein politisches Denken zu eindimensional. Weizen und Holz, so belehrte er Burckhardt, seien die Rohstoffe, mit denen sich das Reich durch Landnahme im Osten eindecken müsse. In dieser rudimentären Volkswirtschaftslehre war für subtilere Formen des modernen Kapitalismus kein Platz ${ }^{22}$. Er jonglierte mit den demographischen und territorialen Statistiken der Flächenstaaten und Kolonialreiche, wie etwa bei seiner Rede in Sonthofen ${ }^{23}$, um die Ansprüche Deutschlands zu rechtfertigen. Für Ribbentrop und die deutsche Führung bestand der einzige Zweck des sogenannten Konsultativabkommens vom 6. Dezember darin, mit der ausgesprochenen Anerkennung der deutsch-französischen Grenze, sprich dem Verzicht auf Elsaß-Lothringen, das unausgesprochene Desinteresse Frankreichs an Osteuropa einzuhandeln. So wie das vorgespielte Interesse an der Rückgabe deutscher Kolonien die britische Regierung zur Räson bringen sollte, zur Konzentration aufs Empire, verbunden mit einer No-Intervention-Politik auf dem Kontinent, wie zu Zeiten der ersten Reichsgründung, so sollte Paris

${ }^{19}$ S. Rainer Blasius, Für Großdeutschland gegen den großen Krieg. Staatsseketär Ernst Freiherr von Weizsäcker in den Krisen um die Tschechoslowakei und Polen 1938/39 (Köln 1981). ${ }^{20}$ Hans F. Bellstedt, "Apaisement" oder Krieg. Frankreichs Außenminister Georges Bonnet und die deutsch-französische Erklärung vom 6. Dezember 1938 (Bonn 1993).

${ }^{21}$ Anthony Adamthwaite, The Franco-German Declaration of 6 December 1938, in: Les Relations Franco-Allemandes 1933-1939, Strasbourg 1-10 octobre 1975, hrsg. v. Centre Nationale de la Recherche Scientifique (Paris 1976) 408.

22 Vgl. Burckbardt, $341 \mathrm{f}$.

${ }^{23}$ Siehe Adolf Hitlers Geheimrede vom 23. November 1937 auf der Ordensburg Sonthofen im Allgäu, abgedruckt bei Picker, 481-490. 
die Besitzstandsgarantie für Elsaß-Lothringen wichtiger sein als die Zukunft Böhmens und Mährens ${ }^{24}$. Der Verzicht auf die früheren Reichslande war im Laufe der Zeit in immer drohenderem Ton ausgesprochen worden, nach dem Motto „Wir können auch anders!“ Das „mißverstandene Desinteressement Frankreichs“ an Osteuropa, eine Formulierung Paul Schmidts, rührte daher, daß Ribbentrop die Vorbehaltsklausel im Hinblick auf dritte Mächte nur auf das Verhältnis der Entente-Mächte und auf die Achse Berlin-Rom bezog, während Bonnet offenbar das gesamte französische Bündnissystem darunter verstand 25 . Immerhin zeigt die Vereinbarung vom 6. Dezember, daß Paris zu einer eigenen Politik des apaisement fähig war und nicht nur im Schlepptau der britischen Politik mitgezogen wurde. Mehr noch, es läßt erkennen, daß auch Frankreich die dominierende Rolle des Reiches in Osteuropa anzuerkennen bereit war. Aber eben nur dies, und nicht die Wiederaufnahme des Germanenzuges nach Osten.

Das eigentliche Problem sehe ich darin, daß es bis zum Einmarsch deutscher Truppen in Prag weder in Großbritannien noch in Frankreich einen gesellschaftlichen Konsens für eine konsequente Abschreckungspolitik gegenüber HitlerDeutschland gab. Die Reaktion auf die Herausforderung eines dynamisch auftretenden Regimes war konventionell und defensiv, wenn auch nicht defätistisch. Churchill war in den 1930er Jahren mit seinen ständigen Mahnungen ein politischer Außenseiter. Auch die britische Presse war von der Regierung auf eine konziliante Haltung gegenüber Deutschland eingeschworen worden ${ }^{26}$; das galt insbesondere für die TIMES, die im Ausland als halbregierungsoffizielles Organ eingestuft wurde. Hinzu kam, daß es kein wirklich abschreckendes Waffensystem gab, vergleichbar den Nuklearwaffen der 1950 er Jahre. Oder richtiger gesagt: Die Gefahr eines Bombenkrieges beeindruckte Chamberlain bei weitem mehr als Hitler und war ein weiteres Argument für den verzweifelten Versuch, den deutschen Diktator durch Konzilianz von einer abenteuerlichen Politik abzuhalten. Die britischen Stabschefs hatten der Produktion von Abfangjägern Priorität gegenüber dem weiteren Aufbau der Bomberflotte eingeräumt, nicht zuletzt, weil die Furcht in weiten Kreisen der Bevölkerung vor dem Zerstörungspotential moderner Bomber geradezu obsessiven Charakter angenommen hatte ${ }^{27}$. Weder die Royal Navy noch die hinter den Maginot-Befestigungen verschanzten französischen Truppen vermochten Hitler davon zu überzeugen, daß ihm vom Westen ernsthafte Gefahr drohte, wenn er im Osten „blitzartig“" zuschlug. Nur Rußland konnte ihm gefährlich werden. Daß Ribbentrop der westlichen Diplomatie mit einem Abkommen zuvorgekommen ist, war gewiß der größte Fehlschlag der Entente-Mächte.

${ }^{24}$ Vgl. Lothar Kettenacker, Nationalsozialistische Volkstumspolitik im Elsaß (Stuttgart 1973) 32-44.

25 Vgl. Bellstedt, 43-49.

${ }^{26}$ Siehe Richard Lockett, Twilight of Truth. Chamberlain, Appeasement and the Manipulation of the Press (London 1989).

27 Dazu Uri Bialer, The Shadow of the Bomber. The Fear of Air Attack and British Politics 1932-1939 (London 1980). Man rechnete mit mehreren Hunderttausend Toten in den ersten Kriegswochen. Tatsächlich hatte England am Ende des Krieges 30000 Ziviltote zu beklagen. 
D. C. Watt meint am Ende seines voluminösen Buches über den Ausbruch des Krieges, daß Hitler nur durch einen Staatsstreich der dazu am wenigsten konditionierten Generäle oder durch ein Attentat von seinem Vorhaben hätte abgebracht werden können ${ }^{28}$. Wenn er damit Recht hätte, durfte der Adressat der westlichen Politik nicht ein ideologisch verblendeter und zugleich opportunistisch taktierender Diktator sein, auch nicht Göring als vermeintlicher Exponent deutscher Wirtschaftsinteressen, sondern in erster Linie die deutsche Generalität, von der erwartet werden konnte, daß sie dem Reich keinen Zwei-Fronten-Krieg, aber auch keinen zermürbenden, alle Ressourcen aufzehrenden Ermattungskrieg zumuten würde. Die westlichen Regierungen haben indes weder die durchaus nicht kriegsbegeisterte deutsche Generalität noch potentielle Attentäter ermutigt, indem sie die von Hitler ausgehende Kriegsgefahr propagandistisch groß herausstellten.

Die Franzosen konnten mit einem Zwei-Fronten-Krieg drohen, die Briten mit einem Ermattungskrieg. Angesichts der Festungsmentalität des französischen Generalstabs und erst recht angesichts des Ribbentrop-Molotow-Paktes war es mit dem Bedrohungspotential Frankreichs nicht mehr weit her. Insofern ging von Großbritannien die größere Bedrohung aus: Die Invasion der Insel war ein bei weitem schwierigeres Unternehmen als die Überwindung der Maginot-Befestigungen. Und hinter Großbritannien standen das maritime Empire und eventuell auch die U.S.A. Nie aber hat es Chamberlain vor dem Krieg für nötig gefunden, mit aller Deutlichkeit auf diese Zusammenhänge hinzuweisen. Erst nach der Kriegserklärung ließ das War Cabinet am 9. September verlauten, daß man bei den Mobilisierungsmaßnahmen mit einer Kriegsdauer von wenigstens drei Jahren rechne ${ }^{29}$ : also nicht Blitzkrieg, wie es Hitler vorschwebte, sondern ein war of attrition, auf den die Wehrmacht nicht eingestellt war.

Mitunter ist man versucht zu glauben, daß die politischen Inszenierungen eines auf Wildwestfilme spezialisierten Hollywoodregisseurs der Situation angemessener gewesen wären als die korrekte Vorgehensweise eines Sir Alexander Cadogan. Gewiß war Botschafter Nevile Henderson, der seine ganze Hoffnung auf den angeblich friedliebenden Göring setzte und stets davor warnte, den deutschen Diktator zu provozieren, der falsche Mann in Berlin. Churchill hätte einen "rude duke "30 nach Berlin geschickt, so wie er dies später für den Moskauer Botschafterposten empfehlen sollte.

${ }^{28}$ Donald C. Watt, How War Came. The Immediate Origins of the Second World War 19381939 (London 1989) 624.

29 The Times vom 11. 9. 1939. Gleichzeitig jedoch prophezeite Chamberlain dem amerikanischen Botschafter ein Ende des Krieges für das kommende Frühjahr, FRUS, 1939/I (Washington 1956) 527. Mit anderen Worten, der britische Premier wußte immer noch nicht, worauf er sich eingelassen hatte.

30 Ursprünglich ein Vorschlag des Northern Department des Foreign Office, dem sich Churchill später anschloß. S. Gabriel Gorodetsky, Stafford Cripps' Mission to Moscow 1940-1942 (Cambridge 1984) xiii und 37. 
Die Machtmittel des britischen Empire reichten aus, den Krieg im Verein mit anderen Mächten als Frankreich zu gewinnen, sie reichten nicht aus, ihn gemeinsam mit Frankreich zu verhindern. Wenn dem Machtanspruch die Machtmittel fehlen, dann gibt es, recht besehen, nur zwei Alternativen: entweder der Verzicht auf ein entscheidendes Mitspracherecht bzw. "menacing silence“ oder ein propagandistisches Trommelfeuer, verbunden mit einer aggressiven Diplomatie. Für keine dieser beiden Alternativen hat man sich in Whitehall entscheiden können, stattdessen die halbherzige Garantie Polens, die das Land Hitler schutzlos auslieferte. 
